\title{
Dynamically generated dimension reduction and crossover in a spin-orbital model
}

\author{
Theja N. De Silva, ${ }^{1,2}$ Michael Ma, ${ }^{1,3}$ and Fu Chun Zhang ${ }^{1,4}$ \\ ${ }^{1}$ Department of Physics, University of Cincinnati, Cincinnati, Ohio 45221, USA \\ ${ }^{2}$ Department of Physics, University of Ruhuna, Matara, Sri Lanka \\ ${ }^{3}$ Department of Physics, Hong Kong University of Science and Technology, Hong Kong \\ ${ }^{4}$ Department of Physics, University of Hong Kong, Hong Kong
}

(Received 9 July 2004; published 17 September 2004)

\begin{abstract}
We study a spin-orbital model in which the spin-spin interaction couples linearly to the orbital isospin. Fluctuations drive the transition from a paramagnetic state to a $C$-type-ordered state into a strongly first-order one, as observed in $\mathrm{V}_{2} \mathrm{O}_{3}$. At $T=0$, there is a ferro-orbital- $C$-spin to ferro-orbital- $G$-spin transition. Close to the transition point, the system shows dynamically generated dimension reduction and crossover, resulting in one or more spin reentrant transitions.
\end{abstract}

DOI: 10.1103/PhysRevB.70.100405

PACS number(s): 75.10. $-\mathrm{b}, 75.25 .+\mathrm{z}, 75.30 . \mathrm{Kz}$

Recently, there has been growing interest in the effects of orbital degeneracy on the physics of transition-metal oxides. The effective spin Hamiltonian of the insulating phase of such systems may depend crucially on orbital short- and long-ranged correlations. As a result, magnetic ordering can become anomalous or may even be suppressed altogether. At the same time, orbital physics is also affected by spin fluctuations and correlations. The interplay between spin and orbital degrees of freedom is fundamental to much of the physics of transition-metal oxides. ${ }^{1,2}$ In this rapid communication we investigate certain aspects of this interplay, emphasizing on the effects of thermal and quantum spin fluctuations on orbital ordering and the effects of orbital ordering on spin physics. Our most interesting result is that the system can exhibit dimension reduction and dimension crossover of spin physics as a function of temperature due to orbital ordering.

In the insulating phase of transition-metal oxides, the dominating energy scales for the transition-metal ions are the on-site Coulomb repulsion, Hund's rule coupling, and the crystal field due to the surrounding oxygen ions. Neglecting weak spin-orbit effect, the general spin Hamiltonian with twofold-degenerate orbitals (represented by pseudospin $\tau$ $=1 / 2)$ is of the form, ${ }^{3} H=\sum_{\langle i j\rangle}\left(J_{i j} \mathbf{S}_{i} \cdot \mathbf{S}_{j}+K_{i j}\right)$, where $J_{i j}$ and $K_{i j}$ are functions of $\tau_{i}$ and $\tau_{j}$. This Hamiltonian has global $\mathrm{SU}(2)$ invariance in spin space and a lower and discrete rotational symmetry in $\tau$ space. $^{4-8}$ Out of the general class of such Hamiltonians, we will focus on those on a cubic lattice of the form

$$
H=J_{0} \sum_{\langle i j\rangle} \mathbf{S}_{i} \cdot \mathbf{S}_{j}-K \sum_{\langle i j\rangle} \mathbf{S}_{i} \cdot \mathbf{S}_{j}\left(\tau_{i} \cdot \hat{\mathbf{n}}_{i j}+\tau_{j} \cdot \hat{\mathbf{n}}_{i j}\right) .
$$

Here, the unit vectors $\hat{\mathbf{n}}_{i j}=\hat{\mathbf{n}}_{1}, \hat{\mathbf{n}}_{2}, \hat{\mathbf{n}}_{3}$ for $i, j$ nearest neighbor in the $x, y, z$ directions, respectively. In this model, the interplay between spins and orbitals arises from the second term which is linear in $\tau$. This linear term will be present provided the two eigenvalues of the hopping matrix are different, while the $\hat{\mathbf{n}}_{i}$ 's will depend on how the two degenerate orbitals transform under lattice rotations. For specificity, we take the $\hat{\mathbf{n}}_{3}$ 's to be unit vectors in the $x-z$ plane, with $\hat{\mathbf{n}}_{3}=\hat{z}$, while $\hat{\mathbf{n}}_{1}$ and $\hat{\mathbf{n}}_{2}$ are rotated from $\hat{\mathbf{n}}_{3}$ by $120^{\circ}$ and $240^{\circ}$. More generally, there are also quadratic in $\tau$ terms, which we assume to be weak compared to the two terms kept. This implies that any orbital ordering in the system will be due to spin-orbital coupling rather than the Jahn-Teller effect. Assuming this is the case, and with the choice of $\hat{\mathbf{n}}_{i}$ 's above, this spin-orbital Hamiltonian can serve as a model for one electron or hole per site in the doubly degenerate $e_{g}$ levels of cubic perovskites, ${ }^{4}$ as well as a possible model for $\mathrm{V}_{2} \mathrm{O}_{3} \cdot{ }^{9}$ For the latter, each site on the cubic lattice is the topological equivalence of a vertical pair of sites on the corundum lattice of $\mathrm{V}_{2} \mathrm{O}_{3}$. The coupling $J_{0}$ depends strongly on and decreases with the Hund's coupling, while $K$ is only weakly dependent on it. We consider $J_{0}>0$, and with an appropriate definition of $\tau$, we also have $K>0$. The calculation shown here will be for $S=2$, the value of $S$ for the $\mathrm{V}_{2} \mathrm{O}_{3}$ bond model, but the results are qualitatively the same for other $S$. The results are also applicable to other lattices and other choices of the $\hat{\mathbf{n}}_{i}$ 's.

While $J_{0}$ favors conventional ( $G$-type) antiferromagnetic (AF) correlations so that nearest-neighbor spins are all AF correlated, $K$ favors, along with orbital ordering, anomalous magnetic correlations that break the cubic lattice rotational symmetry, for example, $C$-type ordering with AF correlation in the $a b$ plane and ferromagnetic (FM) correlation in the $c$ direction. In this paper, we investigate the phase diagram of this model in the temperature $T$ and $J_{0} / K$ plane. Our main results are as follow: (1) At low $T$, orbital ordering gives rise to effective dimension reduction of the spin physics for $J_{0} / K$ close to 2. (2) The weakening of orbital ordering with increasing $T$ can lead two dimension crossover from twodimensional (2D) to three-dimensional (3D) and vice versa. (3) The dimension crossover effect together with thermal fluctuation effects on the spins can lead to an order by disorder mechanism and one or more reentrant transitions. (4) The strongly first-order nature of the magnetic transition in $\mathrm{V}_{2} \mathrm{O}_{3}$ is explained. ${ }^{10}$ The underlying physics behind these results are orbital ordering coupled with spin short-ranged correlations and quantum fluctuations.

Within the context of the bond model for $\mathrm{V}_{2} \mathrm{O}_{3}$, our model has been studied by Joshi et al. ${ }^{6}$ using a single-site mean-field theory. In order to include short-range spin correlation and quantum fluctuations, we use a modified mean- 
field approach to decouple the spin-orbital Hamiltonian. We begin with the Bogolyubov-Peierls variational theorem, ${ }^{11}$

$$
F \leqslant F_{0}+\left\langle H-H_{0}\right\rangle .
$$

Here, $F$ is the true free energy of the system and $H$ is the actual Hamiltonian given in Eq. (1). $H_{0}$ is a variational Hamiltonian and \langle\rangle$_{0}$ is the thermal expectation value with respect to $H_{0} . F_{0}$ is the free energy of the system with Hamiltonian $H_{0}$. We take our variational Hamiltonian as

$$
\begin{gathered}
H_{0}=H_{S}+H_{\tau}, \\
H_{S}=J_{\perp} \sum_{\langle i j\rangle}^{z} \mathbf{S}_{i} \cdot \mathbf{S}_{j}+J \sum_{\langle i j\rangle}^{x, y} \mathbf{S}_{i} \cdot \mathbf{S}_{j}, \\
H_{\tau}=-2 K A \sum_{i} \tau_{i z},
\end{gathered}
$$

where $J_{\perp}=J_{0}-2 K t$ and $J_{\|}=J_{0}+K t$, with $t \geqslant 0$. In $H_{S}$ above, the first sum is for nearest-neighbor pairs along the $z$ direction and the second sum is for pairs on the same $x y$ plane. $t$ and $A$ are variational parameters. The forms of $J_{\perp}$ and $J_{\|}$are based on the expectation that orbital ordering will be ferroorbital.

Minimization of free energy with respect to the variational parameters gives two self-consistent equations, $A$ $=\Delta B$ and $t=\left\langle\tau_{z}\right\rangle=\tanh (2 K \beta A) . \quad \Delta B=B_{\perp}-B_{\|}$, with $B_{\perp}$ $=\left\langle\mathbf{S}_{i} \cdot \mathbf{S}_{j}\right\rangle_{z}$ and $B_{\|}=\left\langle\mathbf{S}_{i} \cdot \mathbf{S}_{j}\right\rangle_{x, y}$ as out-of-plane and in-plane nearest-neighbor spin-spin correlation obtained from $H_{S}$. Thus, the variationalapproach is equivalent to a mean-field decoupling of $\mathbf{S}_{i} \cdot \mathbf{S}_{j} \tau_{i} \rightarrow\left\langle\mathbf{S}_{i} \cdot \mathbf{S}_{j}\right\rangle \tau_{i}+\mathbf{S}_{i} \cdot \mathbf{S}_{j}\left\langle\tau_{i}\right\rangle-\left\langle\mathbf{S}_{i} \cdot \mathbf{S}_{j}\right\rangle\left\langle\tau_{i}\right\rangle$. Note that while a nonzero value of $t$ signifies long-range orbital order, a nonzero value of $B$ only signifies short-range spin correlations. If $B_{\perp} \neq B_{\|}$, spin correlations will be different from isotropic $G$-type antiferromagnet. In particular, if $B_{\|}<0$, and $B_{\perp}>0$, that would correspond to $C$-type magnetic correlations. Our decoupling scheme thus allows us to study the effects of short-ranged spin-spin correlations, but not short-ranged orbital correlations. However, we expect spin fluctuations to be dominant because of its continuous symmetry. Joint spin-orbital correlations are also ignored, but we expect these to be weak compared to spin fluctuations far away from the SU(4) limit. ${ }^{12}$ Based on these arguments, our choice of $H_{0}$ should provide a good approximation for the relevant physics.

Note that $J_{\|}>0$, but $J_{\perp}$ can be either positive or negative in $H_{S}$, depending on the value of orbital order parameter $t$. $H_{S}$ is an Heisenberg Hamiltonian with spatial anisotropy. In order to include short-ranged correlations and quantum fluctuations, we use renormalized spin-wave theory (RSWT) ${ }^{13-15}$ which, unlike traditional spin-wave theory (SWT), is applicable to both a magnetically ordered and disordered phase. For the Heisenberg antiferromagnet on the square lattice, Hirsch and Tang ${ }^{14}$ have shown that this method can provide quantitatively accurate results. In RSWT, magnon-magnon interactions are approximated by introducing a constraint that the total staggered magnetization be zero; $M=\sum_{i \in A} S_{i}^{z}-\sum_{j \in B} S_{j}^{z}=0$, or equivalently, that the average number of spin waves per site is $S$. This constraint can be implemented by introducing into $H_{S}$ a Lagrange multiplier $\lambda$,

$$
H_{S}=J_{\perp} \sum_{\langle i j\rangle}^{z} \mathbf{S}_{i} \cdot \mathbf{S}_{j}+J \sum_{\langle i j\rangle}^{x, y} \mathbf{S}_{i} \cdot \mathbf{S}_{j}-\lambda M .
$$

The modified $H_{S}$ [Eq. (4)] is then solved using the usual spin-wave theory by expanding to quadratic order in Holstein-Primakoff bosons.

Classically, the spins will order as $C$ and $G$ type for the $J_{\perp}<0$ and $J_{\perp}>0$ cases, respectively. Thus, the sublattice designation will differ in the two cases and the spin-wave calculation must be done separately. Let us define $Q=J_{\perp} / J_{\|}$ and $\omega_{k}^{+}$and $\omega_{k}^{-}$, magnon energies for $Q>0$ and $Q<0$ cases, respectively. We see that $Q$ is a dimensionless measure of the effective spin-spin coupling anisotropy. Note that $-2<Q<1$. The chemical potential $\mu$ is obtained from the constraint equation,

$$
S+\frac{1}{2}=\int \frac{d^{d} \vec{k}}{(2 \pi)^{d}}\left(\frac{1}{e^{\beta \omega_{k}^{ \pm}}-1}+\frac{1}{2}\right) F_{ \pm}(\mu, k),
$$

where $\beta$ is the inverse temperature and $\omega_{k}^{ \pm}$are given by $\omega_{k}^{+}=6 J_{\|} S \sqrt{\mu^{2}-\gamma_{k}^{2}}$ and $\omega_{k}^{-}=6 J_{\|} S \sqrt{\left[\mu-(|Q| / 3) \gamma_{k \perp}\right]^{2}-\left[\frac{2}{3} \gamma_{k \|}\right]^{2}}$ with effective magnon chemical potential, $\quad \mu=\frac{1}{3}[2+|Q|$ $+\left(\lambda / 4 S J_{\|}\right) \cdot \gamma_{k}=\frac{1}{3}\left(\cos k_{x}+\cos k_{y}+Q \cos k_{z}\right), \gamma_{k \perp}=\cos k_{z}$, and $\gamma_{k \|}=\frac{1}{2}\left(\cos k_{x}+\cos k_{y}\right) . F_{+}(\mu, k)=\mu\left(\mu^{2}-\gamma_{\vec{k}}^{2}\right)^{-1 / 2}$ and $F_{-}(\mu, k)$ $=\left[\mu+(Q / 3) \gamma_{k \perp}\right]\left[\left(\mu+(Q 3) \gamma_{k \perp}\right)^{2}-\left(\frac{2}{3} \gamma_{k \mid}\right)^{2}\right]^{-1 / 2}$ should be used for $Q>0,<0$, respectively.

After solving for $\mu, \Delta B$ can be calculated, and the selfconsistent equation for $t$ can be solved using an iterative scheme. When there is more than one solution for $t$, we compare their free energies to choose the stable solution for each temperature. One anomaly of the RSWT approach is that $B_{\perp}$ and $B_{\|}$need to be calculated to one order of $1 / S$ higher than the free energy to ensure the correct sign for spin correlation at high temperatures. ${ }^{13,16}$ This difference is not significant for the self-consistent solutions close to and below the temperature of the first phase transition in our model.

In Fig. 1, we show the resulting phase diagram for the case of $S=2$, the value of $S$ for the $\mathrm{V}_{2} \mathrm{O}_{3}$ bond model. In what follows, P stands for para (i.e., disordered), F stands for ferro-ordering, $\mathrm{C}$ stands for $C$-type ordering, $\mathrm{G}$ stands for $G$-type ordering, and $\mathrm{O}$ and $\mathrm{S}$ refer to orbital and spin, respectively. Five phases are possible in the model. These phases are POPS, FOCS, FOPS (may be GS or CS shortranged correlations), POGS [isotropic AF long-range order (AFLRO)] and FOGS (anisotropic AFLRO). The phase consistent with the magnetic and orbital ordering observed in $\mathrm{V}_{2} \mathrm{O}_{3}$ is the FOCS phase. ${ }^{9,10,17,18}$ The phase transitions that the system undergoes as the temperature is lowered depend on the parameter $J_{0} / K$ and can be grouped into six regimes discussed below. In all cases, the orbital transition is first order while the spin transitions are second order, unless accompanied by the orbital transition.

For regime I $\left[0 \leqslant J_{0} / K \leqslant 0.506\right]$, the regime relevant for $\mathrm{V}_{2} \mathrm{O}_{3}$, the transition is a direct one from the disordered phase into the FOCS phase with both spins and orbitals ordered. 

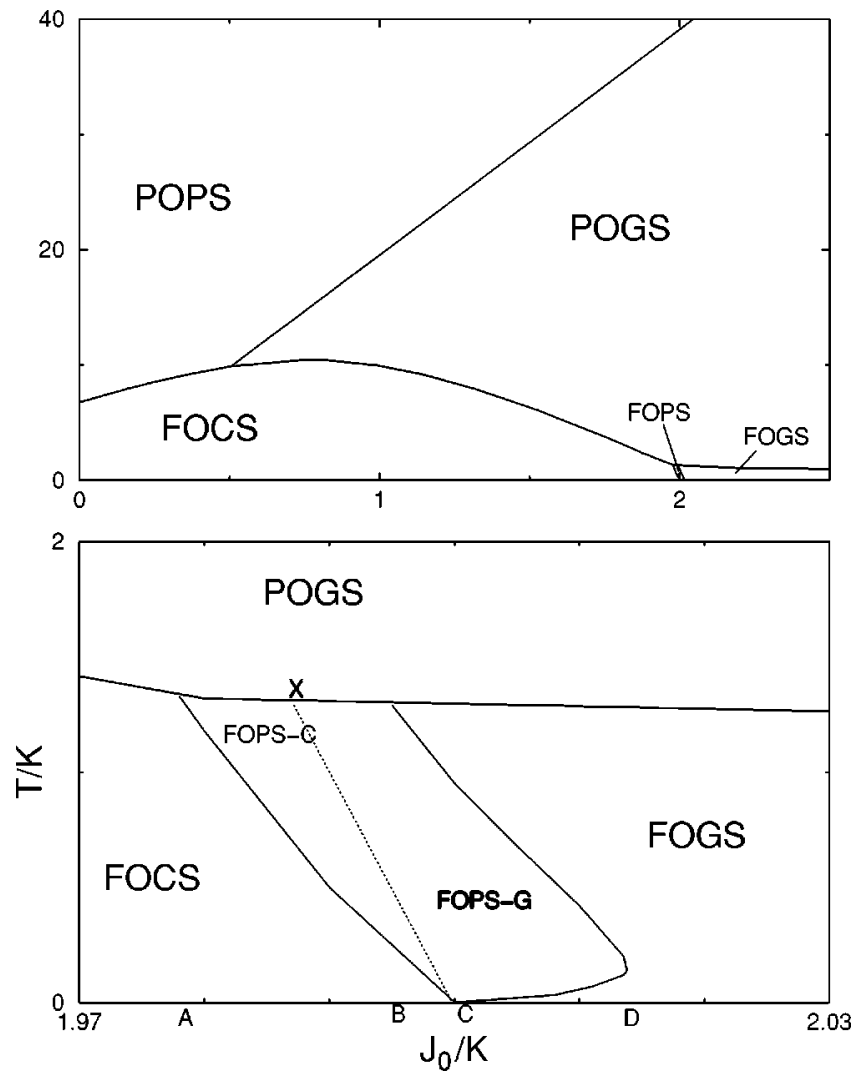

FIG. 1. (a) Phase diagram of spin-2 doubly degenerated spinorbital model. (b) Blowup of the region close to $J_{0} / K=2$. Regimes III, IV, and $\mathrm{V}$ described in the text correspond to values of $J_{0} / K$ between $\mathrm{AB}, \mathrm{BC}$, and $\mathrm{CD}$, respectively. The dotted line gives $T_{2}$, the dimension reduction temperature. To its left (right), the spin coupling anisotropy parameter $Q<0(>0)$.

Experiments showed this transition to be strongly first order. While single-site mean-field theory (SSMFT) for our Hamiltonian is able to obtain a first-order transition in this parameter regime, it was only weakly first order. By including short-ranged correlations in the present theory, the POPS phase is stabilized, and the first-order nature of the transition is significantly enhanced. Entropy jump calculated from the free-energy derivative is larger than $1 k_{B}$ per site throughout regime I, with, e.g., a value of $1.28 k_{B}$ for $J_{0} / K=0.25$, which compares favorably with the experimental value of $1.4 k_{B}$ (each site on the bond model corresponds to a pair of $\mathrm{V}$ ions). We should note that the mechanism for a large entropy jump here is quite different from the usual fluctuation driven first-order transition, where the dominant fluctuations are from modes close to the mean-field free-energy minimum. For this system, however, the dominant fluctuations are $G$-type magnetic ones, and very far from the $C$-type magnetic ordering. Another issue in $\mathrm{V}_{2} \mathrm{O}_{3}$ is why spins and orbitals order at the same temperature. In principle, it is possible to have a spin Peierls transition driven by FO ordering instead of a FOCS transition. ${ }^{6}$ This issue cannot be addressed by SSMFT, but can be using our modified MFT, which shows that for $J_{0} / K$ appropriate for $\mathrm{V}_{2} \mathrm{O}_{3}$, there is no $\mathrm{FO}$ driven spin Peierls phase. However, we will see below that such a phase can indeed occur in other parameter regimes of our model, but it will necessarily be preceded by a POGS ordering at higher temperature. Thus, a phenomenological explanation of the concurrence of orbital and CS ordering in $\mathrm{V}_{2} \mathrm{O}_{3}$ is that there is no GS ordering at a higher temperature.

Regimes II and VI $\left(0.506<J_{0} / K \leqslant 1.976\right.$ and $2.013<J_{0} / K$, respectively) show two phase transitions. The system first undergoes an isotropic $(Q=1)$ POGS ordering. Then, at a lower temperature, the spin-orbital coupling causes a first-order FO transition that converts the spin ordering to $\mathrm{CS}(Q<0)$ in regime II and anisotropic GS $(Q>0, \neq 1)$ in regime VI. Rather more interesting, however, are regimes III, IV, and $\mathrm{V}$, corresponding to $1.976<J_{0} / K$ $\leqslant 1.987,1.987<J_{0} / K \leqslant 2$, and $2<J_{0} / K \leqslant 2.013$, respectively. These regimes show multiple transitions, including reentrance. These transitions are consequences of effective dimension reduction and dimension crossover in spin physics caused by orbital ordering.

To see this, let us first consider $T=0$, where within our MFT the orbital is always fully ordered. As a result, the effective spin Hamiltonian from our decoupling scheme parametrized by $Q$ changes continuously to smaller positive $Q$ and then eventually to negative $Q$ as $J_{0} / K$ is decreased. At $J_{0} / K=2, Q=0$ and the spins on different planes become decoupled, i.e., the spin Hamiltonian is that of a 2D Heisenberg antiferromagnet. This is the orbital driven dimension reduction effect at $T=0$. Current wisdom is that the ground state of the 2D Heisenberg antiferromagnetic Hamiltonian is ordered at $T=0$ in $2 \mathrm{D}$ even for $S=1 / 2$. Thus, there is spin LRO (anisotropic GS or CS) for all values of $J_{0} / K$ at $T=0$. At finite temperature, thermal fluctuations will weaken both the spin ordering and the orbital ordering, with the latter giving rise to effective temperature-dependent spin Hamiltonian. The spin physics is best understood by considering how $Q(t)$ changes with $T$ together with the dependence of the spin transition temperature $T_{c}(Q)$ on $Q$. For small $|Q|, T_{c}(Q)$ $\sim|Q|^{\theta}$ RSWT gives $\theta=1 / 2$. Because orbital has a discrete symmetry, its order parameter $t$, and hence $Q$ changes exponentially slowly at low $T$, and the physics is dominated by thermal disordering of the spin. At higher $T$, the reduction in $t$ becomes significant, and the corresponding change in $Q$ can give rise to dimension crossover in spin physics. These features are shown in Fig. 1(b) and discussed below, where $Q_{0}$ denotes the value of $Q$ for $t=1$, i.e., at $T=0$.

We first consider $J_{0} / K=2$ right at the decoupling point [point $\mathrm{C}$ in Fig. 1(b)], so that $Q_{0}=0$ at $T=0$. As $T$ increases, $t$ decreases and $Q$ becomes increasingly positive, and the planes become increasingly coupled, implying a crossover from $2 \mathrm{D}$ to $3 \mathrm{D}$. However, this crossover is exponentially slow at low $T$, and since Heisenberg spins cannot order at any $T>0$ in two-dimensions, the spin LRO is immediately destroyed at infinitesimal $T$. As temperature increases, $Q$ becomes large enough that $T_{c}(Q)$ exceeds $T$, and there is a reentrant transition into an anisotropic GS phase. The restoration of spin LRO due to temperature-induced dimension crossover can be viewed as a new kind of order by disorder mechanism. For $Q_{0}>0$ but small (regime V), the physics is basically the same with one difference. Since now $T_{c}\left(Q_{0}\right)>0$, the GS order is stable at low $T$ but will disorder for $T \gtrsim T_{c}\left(Q_{0}\right)$. 
The behavior is even richer for $Q_{0}<0$ but small (regimes III and IV). Now as $T$ increases, $Q$ gets first less negative, becomes 0 at some temperature $T_{2}$, then becomes positive. That is, we have dimension crossovers first from anisotropic $3 \mathrm{D}$ to $2 \mathrm{D}$, and then back to anisotropic $3 \mathrm{D}$ as $T$ increases. Correspondingly, the spins first undergo a transition from CS LRO to CS short-ranged order at $T \approx T_{c}\left(Q_{0}\right)$ The interplane ferromagnetic correlation continues to decrease as $T$ increases, crossing over into GS short-ranged order for $T>T_{2}$ In regime $I V$, there is yet another reentrant transition into anisotropic GS LRO. Throughout regimes III-V, the PS phases have short-ranged spin correlations that are spatially anisotropic and so break the lattice rotational symmetry. In effect, these are orbital driven spin Peierls phases. Eventually, in all these regimes, the system switches back in a firstorder jump back into isotropic GS ordering when the orbital becomes disordered. Point $X$ is where this transition coincides with the dimension reduction temperature $T_{2}$, so amazingly there is a jump directly from isotropic $3 \mathrm{D}$ behavior just above the transition to exactly $2 \mathrm{D}$ just below.

In summary, we have investigated the problem of the interplay between spins and orbitals in transition-metal oxides concentrating on the competition between spin-spin interactions and spin-orbital coupling. In addition to illuminating the phase-transition properties of magnetic ordering in insulating $\mathrm{V}_{2} \mathrm{O}_{3}$, our model shows a mechanism for dynamically generated dimensional reduction and dimension crossover. Although the results presented are for $S=2$, the same qualitative behavior will hold for other $S$. Also, while our calculations are restricted to the Hamiltonian [Eq. (1)], these effects will be present in other spin-orbital models as long as orbital ordering results in vanishing spin-spin coupling in one or more spatial directions. Since these dimensional reduction and crossover effects are present only close to the decoupling point, to observe them one would need to find systems with the appropriate Hund's coupling so as to produce the proper $J_{0} / K$ range. For $S=2$, there is the additional problem that this range is very narrow. Larger range will occur for smaller $S$. Therefore, it will be interesting to search for effective $S=1 / 2$ peroskite transition-metal oxides with double orbital degeneracy and weak Jahn-Teller coupling.

This work was in part supported by DE/FG0301ER45687, the University of Cincinnati for financial support as a URC Summer Student, and by the Chinese Academy of Sciences. We thank Dung-Hai Lee, Pak-Wo Leung, and R. J Gooding for useful discussions.
${ }^{1}$ For a review see, Y. Tokura and N. Nagaosa, Science 288, 462 (2000).

${ }^{2}$ M. Imada, A. Fujimori, and Y. Tokura, Rev. Mod. Phys. 70, 1039 (1998).

${ }^{3}$ K. I. Kugel and D. I. Khomskii, Sov. Phys. JETP 52, 501 (1981).

${ }^{4}$ D. I. Khomskii and M. V. Mostovoy, preprint, cond-mat/0304089 (unpublished).

${ }^{5}$ C. Castellani, C. R. Natoli, and J. Ranninger, Phys. Rev. B 18, 4945 (1978).

${ }^{6}$ A. Joshi, M. Ma, and F. C. Zhang, Phys. Rev. Lett. 86, 5743 (2001).

${ }^{7}$ T. N. De Silva, A. Joshi, M. Ma, and F. C. Zhang, Phys. Rev. B 68, 184402 (2003).

${ }^{8}$ G. Khaliullin, P. Horsch, and A. M. Oles, Phys. Rev. Lett. 86, 3879 (2001).

${ }^{9}$ F. Mila, R. Shiina, F. C. Zhang, A. Joshi, M. Ma, V. Anisimov, and T. M. Rice, Phys. Rev. Lett. 85, 1714 (2000).

${ }^{10}$ W. Bao, C. Broholm, G. Aeppli, S. A. Carter, P. Dai, T. F. Rosen- baum, J. M. Honig, P. Metcalf, and S. F. Trevino, Phys. Rev. B 58, 12727 (1998).

${ }^{11}$ R. P. Feynman, Statistical Mechanics, Frontiers in Physics Series, (Benjamin, New York, 1972); R. Peierls, Phys. Rev. 54, 918 (1938).

${ }^{12}$ Y. Q. Li, M. Ma, D. N. Shi, and F. C. Zhang, Phys. Rev. Lett. 81, 3527 (1998).

${ }^{13}$ M. Takahashi, Phys. Rev. Lett. 58, 168 (1987).

${ }^{14}$ J. E. Hirsch and S. Tang, Phys. Rev. B 40, 4769 (1989).

${ }^{15}$ S. Tang, M. E. Lazzouni, and J. E. Hirsch, Phys. Rev. B 40, 5000 (1989).

${ }^{16}$ M. Takahashi, Phys. Rev. B 40, 2494 (1989).

${ }^{17}$ L. Paolasini, C. Vettier, F. de Bergevin, F. Yakhou, D. Mannix, A. Stunault, W. Neubeck, M. Altarelli, M. Fabrizio, P. A. Metcalf, and J. M. Honig, Phys. Rev. Lett. 82, 4719 (1999).

${ }^{18}$ J. H. Park, L. H. Tjeng, A. Tanaka, J. W. Allen, C. T. Chen, P. Metcalf, J. M. Honig, F. M. F. de Groot, and G. A. Sawatzky, Phys. Rev. B 61, 11506 (2000). 